\title{
Antiferromagnetic resonance in a spin-gap magnet with strong single-ion anisotropy
}

\author{
V.N.Glazkov ${ }^{1, *}$ \\ ${ }^{1}$ P.Kapitza Institute for Physical Problems RAS, 119334 Moscow, Russia
}

(Dated: November 6, 2020)

\begin{abstract}
Quasi-one-dimensional magnet $\mathrm{NiCl}_{2} \cdot 4 \mathrm{SC}\left(\mathrm{NH}_{2}\right)_{2}$, usually abbreviated as DTN, does not order at zero field down to $T=0$ : due to the strong single-ion anisotropy of the "easy plane" type acting on $S=1 \mathrm{Ni}^{2+}$ ions, the $S_{z}=0$ ground state is separated from $S_{z}= \pm 1$ excitations by an energy gap. Once the magnetic field is applied along the main anisotropy axis, the gap closes at $B_{c 1}=2.18 \mathrm{~T}$ and the field-induced antiferromagnetic order arises. The low-energy excitations spectrum of this field-induced ordered state includes two branches of excitations, one of them have to be a gapless Goldstone mode. Recent studies of excitations spectrum in a field-induced ordered state of DTN (T.Soldatov et.al, Phys.Rev.B 101, 104410 (2020)) have revealed that Goldstone mode became gapped as magnetic field deviates from the main symmetry axis. This paper proposes simple description of antiferromagnetic resonance modes of quasi-one-dimensional quantum $S=1$ magnet with strong single-ion anisotropy. The approach used is based on a combination of the strong coupling model for the anisotropic spin chain with the conventional mean-field model of antiferromagnetic resonance. The resulting model fits to the known experimental results without additional tuning parameters.
\end{abstract}

Keywords: spin-gap magnets, magnetic resonance, field-induced ordering

Metal-organic compound DTN $\left(\mathrm{NiCl}_{2} \cdot 4 \mathrm{SC}\left(\mathrm{NH}_{2}\right)_{2}\right)$ is an example of the collective paramagnet with gapped excitations spectrum (spin-gap magnet). Magnetic ions $\mathrm{Ni}^{2+}(S=1)$ form chains running along the four-fold axis of the tetragonal crystal [1, 2]. Contrary to Haldane magnets, magnetic ions in DTN are subjects of the strong single-ion anisotropy, which separates $S_{z}=0$ single-ion state from the excited doublet $S_{z}= \pm 1$. Exchange coupling turns out to be weak as compared to the single ion anisotropy, it leaves ground state nonmagnetic and makes $S_{z}= \pm 1$ excitations delocalized. Applied magnetic field reduces energy of one of the excited states and closes the gap at a certain critical field, leading at the same moment to the formation of the field-induced long-range order due to the weak inter-chain coupling 3 , 4 ].

Field-induced ordering of the spin-gap magnets was actively discussed in the literature in relation with BoseEinstein condensation of magnons $[5,6]$. One of expected properties of the field-induced ordered state is the existence of a gapless Goldstone mode due to the conservation of axial symmetry above the critical field, but the low symmetry of the real magnets can violate this prediction. Tetragonal symmetry of DTN crystals makes this compound one of the most suitable candidates for the search of the Goldstone mode in the field-induced ordered state.

Dynamics of low-energy excitations in DTN was studied by means of electron spin resonance (ESR) spectroscopy in Refs. [7 9]. Recent study by Soldatov et. al. 9] has revealed that at small tilt (up to $5^{\circ}$ ) of the applied field from the four-fold symmetry axis the Goldstone mode becomes gapped. ESR frequencies in the field-induced ordered phase of DTN were calculated us-

\footnotetext{
* glazkov@kapitza.ras.ru
}

ing various theoretical approaches [8, 10], however, these models do not yield compact expressions neither for the magnetic resonance eigenfrequencies nor for the dependencies of the ESR spectra parameters on exchange coupling and single-ion anisotropy constants. This work provides interpretation of the ESR eigenfrequencies in the field-induced ordered phase of the $S=1$ quantum magnet DTN, which allows to follow characteristic dependence of ESR eigenfrequencies on the parameters of microscopic model and describes quantitatively results obtained for the weakly tilted magnetic field.

Spin Hamiltonian for DTN can be written as:

$$
\mathcal{H}_{\text {chain }}=\sum_{i}\left(D S_{z, i}^{2}+J \vec{S}_{i} \vec{S}_{i+1}-g \mu_{B} \vec{B} \vec{S}_{i}\right)
$$

for DTN $D=8.9 \mathrm{~K}, J=2.2 \mathrm{~K}$, strongest inter-chain exchange integral is approximately ten-fold smaller $(0.18 \mathrm{~K})$ and is omitted in (11), the $g$-factor value for the field applied along the tetragonal axis equals $g=2.26$ [7].

In the strong-coupling limit $D \gg J$ dispersion of the spin excitations can be found within conventional perturbations theory for $\vec{B} \| Z$ as long as the applied field is below the critical value. Zero-field excitations spectrum for the $S=1$ case is found up to third order over $J / D$ in Ref. 11] (similar result is also known for the arbitrary spin [12]), magnetic field can be taken into account straightforwardly since $S_{z}$ remains a good quantum number. One-magnon excitations spectrum above the saturation field can be found exactly (Ref. 7] includes these calculations for the case of DTN accounting for the interchain couplings as well).

Minimal energy of these excitations at $(k a)=\pi$ turns 


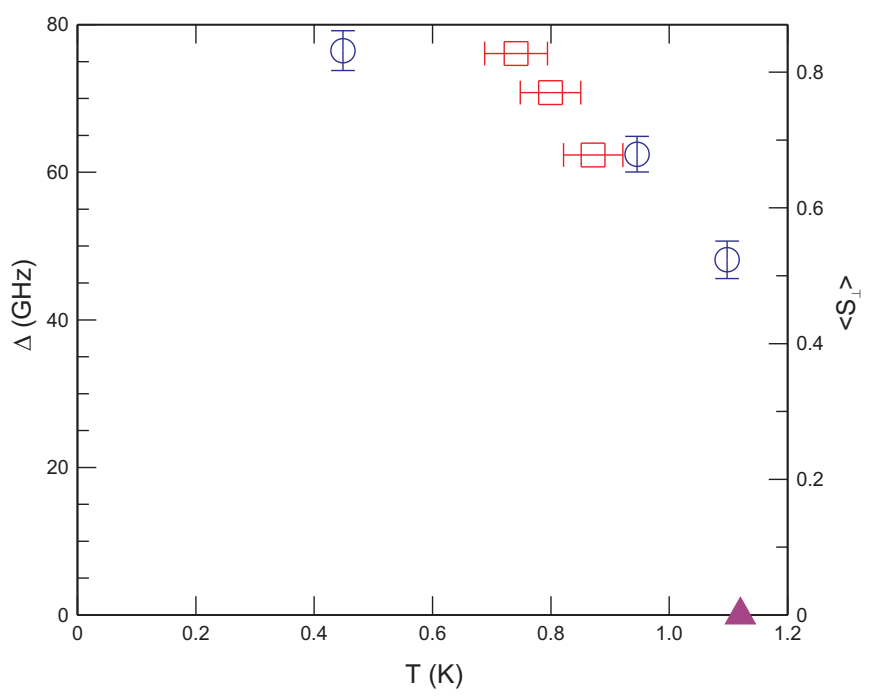

FIG. 1. Temperature dependence of the mean value of the ordered transverse spin component at the field $8 \mathrm{~T},\left\langle S_{\perp}\right\rangle$ values are calculated from experimental data using Eqn. 8 . Left Yaxis shows gap values $\Delta$ from Ref. [8], right Y-axis shows the mean value of the ordered transverse spin component. Open symbols are $\Delta(T)$ data: squares corresponds to the temperature scans at the fixed frequency, circles corresponds to the $f(B)$ measurements at the fixed temperature. Filled triangle on $\mathrm{X}$-axis is the temperature of phase transition at the field of $8 \mathrm{~T}$ according to Ref. [2].

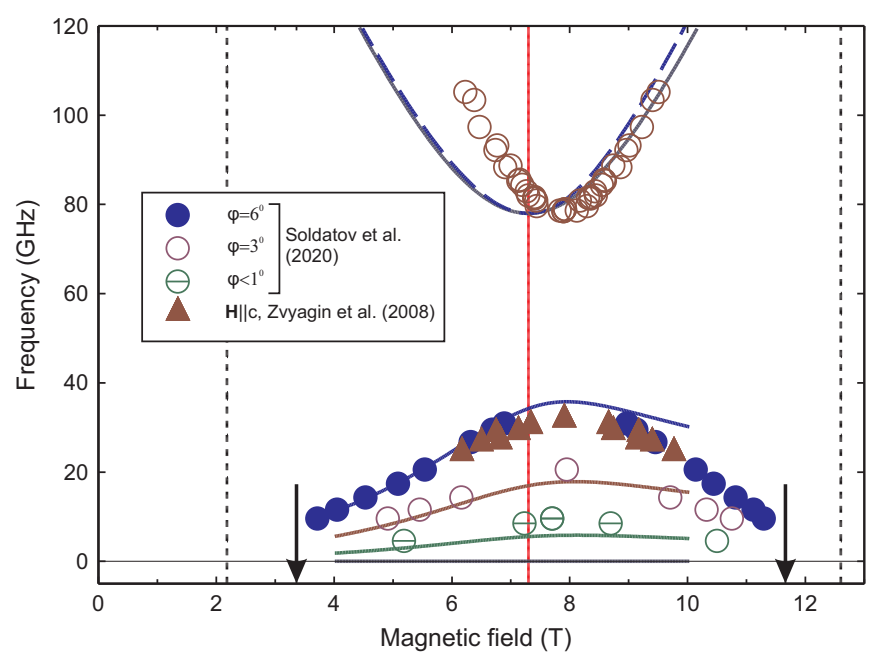

FIG. 2. Comparison of the model results (Eqn. (12)) with the experimental results of Ref. 9] (circles) and Ref. [8] (triangles). Vertical solid line marks $B_{0}$ value, vertical dashed lines mark experimentally found values of the critical fields $B_{c 1}$ and $B_{c 2}$, arrows mark critical field values computed from Eqns. (2), (3). Curves are model results for the Hamiltonian parameters given in the text. Curves for the lower ESR branch corresponds to the tilt angles (bottom to top) $0^{\circ}, 1^{\circ}$, $3^{\circ}$ and $6^{\circ}$, correspondingly. Curves for the upper branch are computed for the tilt angles $0^{\circ}$ (solid curve) and $6^{\circ}$ (dashed curve). to zero at critical fields [7, 13]:

$$
\begin{aligned}
& g \mu_{B} B_{c 1}=D-2 J+\frac{J^{2}}{D}+\frac{J^{3}}{2 D^{2}} \\
& g \mu_{B} B_{c 2}=D+4 J
\end{aligned}
$$

Critical fields $B_{c 1}$ and $B_{c 2}$ are close within the strong coupling limit $D \gg J$. At $B_{c 1}<B<B_{c 2}$ single-ion Hamiltonian can be projected on the two lower states $S_{z}=0$ and $S_{z}=1$. This two-level system can be formally described by a pseudo-spin $T=1 / 2$ by relabeling singleion states: $T_{z}=-1 / 2 \Leftrightarrow S_{z}=0$ and $T_{z}=1 / 2 \Leftrightarrow S_{z}=1$ 13]. Spin operators have to be replaced as follows:

$$
\begin{aligned}
S_{z} & =T_{z}+1 / 2 \\
S^{ \pm} & =\sqrt{2} T^{ \pm}
\end{aligned}
$$

After this substitution Hamiltonian (1) transforms at $\vec{B} \| Z$ into:

$$
\begin{aligned}
\mathcal{H} & =2 J \sum_{i}\left(T_{x, i} T_{x, i+1}+T_{y, i} T_{y, i+1}+\frac{1}{2} T_{z, i} T_{z, i+1}\right)+ \\
& +\left(J+D-g \mu_{B} B\right) \sum_{i} T_{z, i}+N \frac{2 J+D-g \mu_{B} B}{2}
\end{aligned}
$$

this transformation has linear accuracy over $J / D$.

Thus, the problem of the $S=1$ spin chain with strong single-ion anisotropy is mapped onto the equivalent model of uniform $S=1 / 2$ XXZ-chain with strong XY-anisotropy and the effective field $B_{\text {eff }}=$ $B-(J+D) /\left(g \mu_{B}\right)$. Effective field turns to zero at the applied field value $B_{0}=(J+D) /\left(g \mu_{B}\right)$, which is within first order of perturbations theory equal to the half-sum of the critical fields. One can also note that frequencies of the ESR-active transitions at $k=0$ at $B<B_{c 1}$ within this approximation are given by $h f=\left(D+2 J+\frac{J^{2}}{D}-\frac{J^{3}}{2 D} \pm g \mu_{B} B\right)$, extrapolation of the lower frequency turns to zero at $B_{0}^{(\mathrm{PM})}=\left(D+2 J+\frac{J^{2}}{D}-\frac{J^{3}}{2 D}\right) /\left(g \mu_{B}\right)$, the later value is close to $B_{0}$ and $B_{c 1}$ but does not coincides with either of them. Similarly, extrapolation of the high-field ESR frequency $(k=0)$ at $B>B_{c 2}, h f=\left(g \mu_{B} B-D\right)$, turns to zero at the field value $B_{0}^{(\mathrm{HF})}=D /\left(g \mu_{B}\right)$ [7] different from both $B_{0}$ and $B_{c 2}$.

In terms of pseudo-spins inter-chain coupling also transforms into interaction with strong XY-anisotropy. Thus, at $B=B_{0}$ and $T=0$ quasi-one dimensional $S=1$ magnet can be mapped on an equivalent model of the ordered $3 \mathrm{D} S=1 / 2$ antiferromagnet with strong "easy plane" anisotropy in zero effective field.

Once the applied field deviates from $B_{0}$ (but remains parallel to $Z$ ), the equivalent model becomes that of an "easy plane" antiferromagnet in a field $B_{\text {eff }}=B-B_{0}$, applied along the anisotropy axis $Z$. Critical fields $B_{c 1}$ and $B_{c 2}$, symmetrically located to the left and to the right from $B_{0}$, are the saturation fields for the 
equivalent model. ESR eigenfrequencies (antiferromagnetic resonance eigenfrequencies) for the "easy plane" antiferromagnet with strong anisotropy can be found within the conventional sublattices model [14, 15]: for $\vec{B}_{e} f f \| Z$ one of the eigenfrequencies is zero, and the second eigenfrequency depends on the effective field as $f=\sqrt{\left(\gamma B_{e f f}\right)^{2}+\Delta^{2}}$, here $\gamma=g \mu_{B} / h$ is a gyromagnetic ratio. The first mode corresponds to the expected Goldstone mode, and the second mode corresponds to the gapped spectral branch observed in [8, 9] (the gap $\Delta$ equals $78 \mathrm{GHz}$ at $T=0.45 \mathrm{~K}$ ).

Within the sublattices model the gap $\Delta$ can be derived from the $X Y$-anisotropy of the equivalent model (6) and magnitude of the antiferromagnetic order parameter (sublattice magnetization) [13 15]:

$$
\Delta=\gamma \sqrt{2 H_{A} H_{E}}
$$

here $H_{E}=4 J \mu /\left(g^{2} \mu_{B}^{2}\right)$ is the characteristic exchange field, $H_{A}=J \mu /\left(g^{2} \mu_{B}^{2}\right)$ is the characteristic anisotropy field for the equivalent model and $\mu$ is the average sublattice magnetization for the equivalent model, the inter-chain couplings are neglected here. Hence $\Delta=$ $2 \sqrt{2}(J / h)\left\langle t_{\perp}\right\rangle$, here $\left\langle t_{\perp}\right\rangle$ is the mean pseudo-spin projection on the XY-plane. Transforming this result back to the real spins (5) one obtains expression for the gap of the upper ESR branch in terms of transverse component of the real spin

$$
\Delta=\frac{2 J}{h}\left\langle S_{\perp}\right\rangle
$$

Thus, one can conclude that magnitude of the gap $\Delta$ for the upper ESR branch in the ordered phase is mostly determined by inter-chain exchange integral $J$, while the position of the minimum of this branch (field $B_{0}$ ) is mostly determined by the single-ion anisotropy constant D.

Gap $\Delta$ was measured as a function of temperature in Ref. [8]. These data allows to recover temperature dependence of the order parameter $\left\langle S_{\perp}\right\rangle$ at the field of $8 \mathrm{~T}$
(Fig. 1). The result is qualitatively sound: obtained values of the order parameter $\left\langle S_{\perp}\right\rangle<1$.

Now we will allow applied magnetic field to tilt slightly from the $Z$ direction, this tilt towards $X$ axis can be parameterized by an angle $\Theta$. At small tilt angles fieldinduced antiferromagnetic ordering persists, while the critical field are slightly rescaled [16]. Linearly over $\Theta$, Zeeman contribution to the pseudo-spin Hamiltonian (6) becomes:

$$
\mathcal{H}_{Z}=-g \mu_{B} B_{\text {eff }} \sum_{i} T_{z, i}-\sqrt{2} g \mu_{B} B \Theta \sum_{i} T_{x, i}
$$

i.e., additional contribution arises which is proportional to the full applied field and is applied within the "easy plane". This contribution is assumed to be small: $g \mu_{B} B \Theta \simeq D \Theta \ll J \ll D$.

When the applied field is equal to $B_{0}$, the $Z$ component of the effective field is zero and our equivalent pseudo-spin model is that of an "easy plane" antiferromagnet with the field applied within the "easy plane". Eigenfrequencies of such an antiferromagnet [14, 15] are $f_{1}=\gamma B_{\text {in-plane }}$ and $f_{2}=\Delta$. Thus, one obtain quantitative result for the ESR frequency of the lower branch at the field $B_{0} \approx\left(B_{c 1}+B_{c 2}\right) / 2$ :

$$
f_{1}(\Theta)=\sqrt{2} \gamma B_{0} \Theta
$$

Note, that contrary to the interpretation of the gap for the upper ESR branch, the later result is independent from the magnitude of the order parameter in the fieldinduced ordered phase.

If the amplitude of the applied field deviates from $B_{0}$, resulting effective field will be applied at some angle within the $X Z$-plane: $\vec{B}_{\text {eff }}=\left(\sqrt{2} B \Theta ; 0 ;\left(B-B_{0}\right)\right)$ and one can use known expression for the antiferromagnetic resonance eigenfrequencies 14

$$
\frac{\left(\gamma B_{e f f, x}\right)^{2}}{f^{2}}+\frac{\left(\gamma B_{e f f, z}\right)^{2}}{f^{2}-\Delta^{2}}=1
$$

hence, for the resonance frequencies

$$
f_{1,2}^{2}=\frac{1}{2}\left[\Delta^{2}+2(\gamma B \Theta)^{2}+\gamma^{2}\left(B-B_{0}\right)^{2} \pm \sqrt{\left(\Delta^{2}+2(\gamma B \Theta)^{2}+\gamma^{2}\left(B-B_{0}\right)^{2}\right)^{2}-8 \Delta^{2}(\gamma B \Theta)^{2}}\right]
$$

The model above, in fact, relies on a classical meanfield treatment of the two-sublattices antiferromagnet. Within the classical mean-field approach magnetization process is linear in field up to the saturation field. Due to one-dimensionality of DTN magnetization changes nonlinearly approaching the critical fields [4, 6, 7], and, moreover, this nonlinearity is not fully symmetric on ap- proaching $B_{c 1}$ from above and $B_{c 2}$ from below. This asymmetry of the real magnet properties compared to the model predictions is due to the limited accuracy of the linear over $J / D$ approximation used for mapping of the real spin problem (1) on a simpler pseudo-spin problem (6). Thus, the proposed model is applicable to DTN only in certain vicinity of $B_{0}$, the range of model applica- 
bility can be roughly estimated as a field range with linear $M(B)$ dependence, which extends [7] from 4 to $10 \mathrm{~T}$. To go beyond these limits would require both to take into account higher orders on $J / D$ when transforming to pseudo-spin representation, and to take into account one-dimensionality of DTN spin subsystem. However, one can be certain that the frequency of the lower ESR branch will turn to zero at the critical fields $B_{c 1}$ and $B_{c 2}$, which are the saturation fields of the equivalent model.

Comparison of the model curves with experimental data from Ref. [9] is shown at Fig. 2] Model curves are computed without additional fitting parameters using the microscopic Hamiltonian (11) constants $D=8.9 \mathrm{~K}$, $J=2.2 \mathrm{~K}, g=2.26(\gamma=31.6 \mathrm{GHz} / \mathrm{T})$ [7], which yields $B_{0}=7.3 \mathrm{~T}$ (half-sum of experimentally measured values of $B_{c 1}$ and $B_{c 2}$ equals $7.4 \mathrm{~T}$ ), and experimentally measured value $\Delta=78 \mathrm{GHz}[8,9]$. Model curves were computed for the tilt angle $\Theta$ equal to $0^{\circ}, 1^{\circ}, 3^{\circ}, 6^{\circ}$, as was used in the experiment 9] (note that the practical accuracy of tilt angle determination is around $\simeq 1 \ldots 2^{\circ}$ ). One can see, that the model curves agree well with the experimental data.

Summing it up, this paper proposes simple and illustrative model to describe magnetic resonance eigenfrequencies in the field-induced antiferromagnetically ordered phase of the quasi-one dimensional magnet with strong "easy-plane" anisotropy $\mathrm{NiCl}_{2} \cdot 4 \mathrm{SC}\left(\mathrm{NH}_{2}\right)_{2}$. The proposed model provides simple relations between ESR eigenfrequencies and microscopic Hamiltonian parameters, it is also applicable for the case of magnetic field slightly tilted from the main anisotropy axis.

The work was supported by the Russian Science Foundation Grant No. 17-12-01505 and by the Program of RAS Presidium "Actual problems of lowtemperature physics". Author thanks Prof. A.I.Smirnov and Dr. T.A.Soldatov (Kapitza Institute) for numerous fruitful discussions.
[1] Amparo Lopez-Castro and Mary R. Truter, The crystal and molecular structure of dichlorotetrakisthioureanickel, $\left[\left(\mathrm{NH}_{2}\right)_{2} \mathrm{CS}_{4} \mathrm{NiCl}_{2}\right.$, Journal of the Chemical Society, p.1309 (1963)

[2] V. S. Zapf, D. Zocco, B. R. Hansen, M. Jaime, N. Harrison, C. D. Batista, M. Kenzelmann, C. Niedermayer, A. Lacerda, and A. Paduan-Filho, Bose-Einstein Condensation of $\mathrm{S}=1$ Nickel Spin Degrees of Freedom in $\mathrm{NiCl}_{2}$ $4 \mathrm{SC}\left(\mathrm{NH}_{2}\right)_{2}$, Phys. Rev. Lett. 96, 077204 (2006)

[3] Armando Paduan-Filho, Robert D. Chirico, K. O. Joung, and Richard L. Carlin Field-induced magnetic ordering in uniaxial nickel systems: A second example, J. Chem. Phys. 74, 4103 (1981)

[4] A. Paduan-Filho, X. Gratens, and N. F. Oliveira, Jr, Field-induced magnetic ordering in $\mathrm{NiCl}_{2}-4 \mathrm{SC}\left(\mathrm{NH}_{2}\right)_{2}$, Physical Review B 69, 020405(R) (2004)

[5] T. Giamarchi, C. Ruegg and O. Tchernyshyov, BoseEinstein condensation in magnetic insulators, Nature Physics 4, 198 (2008).

[6] Vivien Zapf, Marcelo Jaime, and C.D. Batista, BoseEinstein condensation in quantum magnets, Reviews of Modern Physics 86, 563 (2014).

[7] S. A. Zvyagin, J. Wosnitza, C. D. Batista, M. Tsukamoto, N. Kawashima, J. Krzystek, V. S. Zapf, M. Jaime, N. F. Oliveira, Jr., and A. Paduan-Filho, Magnetic Excitations in the Spin-1 Anisotropic Heisenberg Antiferromagnetic Chain System $\mathrm{NiCl}_{2}-4 \mathrm{SC}\left(\mathrm{NH}_{2}\right)_{2}$, Phys. Rev. Lett. 98, 047205 (2007)

[8] S. A. Zvyagin, J. Wosnitza, A. K. Kolezhuk, V. S. Zapf, M. Jaime, A. Paduan-Filho, V. N. Glazkov, S. S. Sosin, and A. I. Smirnov Spin dynamics of $\mathrm{NiCl}_{2}-4 \mathrm{SC}\left(\mathrm{NH}_{2}\right)_{2}$ in the field-induced ordered phase, Physical Review B 77, 092413 (2008)

[9] T. A. Soldatov, A. I. Smirnov, K. Yu. Povarov, A. Paduan-Filho, and A. Zheludev Microwave dynamics of the stoichiometric and bond-disordered anisotropic $\mathrm{S}=1$ chain antiferromagnet $\mathrm{NiCl}_{2}-4 \mathrm{SC}\left(\mathrm{NH}_{2}\right)_{2}$, Physical Review B 101, 104410 (2020)

[10] Artemiy S. Sherbakov, Oleg I. Utesov Magnon spectrum and electron spin resonance in antiferromagnet with large single-ion easy plane anisotropy, Journal of Magnetism and Magnetic Materials 518, 167390 (2021) arXiv:2004.02170 (2020)]

[11] N. Papanicolaou and P. Spathis Quantum spin-1 chains with strong planar anisotropy, J.Phys.: Condens. Matter 2, 6575 (1990)

[12] A. V. Sizanov and A. V. Syromyatnikov Bosonic representation of quantum magnets with large single-ion easyplane anisotropy, Physical Review B 84, 054445 (2011)x

[13] K. M. Diederix, J. P. Groen, T. O. Klaassen, N. J. Poulis, R. L. Carlin, Magnetic properties of $S=1$ singlet ground state systems in external field, Physica B+C 97, 113 (1979)

[14] T. Nagamiya, K. Yosida and R. Kubo "Antiferromagnetism" Advances in Physics, 4, 1 (1955).

[15] A. G. Gurevich, G. A. Melkov "Magnetization Oscillations and Waves" CRC Press (1996).

[16] V. M. Kalita and V. M. Loktev Toward the Theory of Quantum Phase Transitions in DTN-Type Van Vleck Antiferromagnets, JETP Letters 93, 534 (2011) 